\title{
Consumo de alcohol e incidencia de hipertensión en una cohorte mediterránea: el estudio SUN
}

\author{
Jorge M. Núñez-Córdobaa , Miguel A. Martínez-Gonzáleza, Maira Bes-Rastrolloa, Estefanía Toledoa, \\ Juan J. Beunza y Álvaro Alonso a,b
}

\begin{abstract}
aDepartamento de Medicina Preventiva y Salud Pública. Clínica Universitaria de Navarra. Universidad de Navarra. Pamplona. Navarra. España.

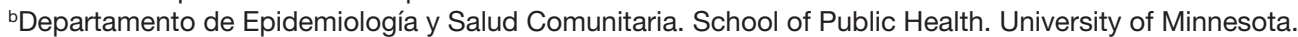

Minneapolis. Minnesota. Estados Unidos.
\end{abstract}

Introducción y objetivos. Evaluar prospectivamente la asociación entre el consumo de alcohol, incluidas la bebida alcohólica preferida y la frecuencia semanal de consumo, y el riesgo de hipertensión en una cohorte mediterránea.

Métodos. Se siguió de manera prospectiva a 9.963 varones y mujeres españoles inicialmente libres de hipertensión. La información recogida sobre dieta y diagnóstico de hipertensión arterial fue la declarada por los pacientes y luego validada.

Resultados. Durante el seguimiento (mediana [intervalo intercuartílico], 4,2 [2,5-6,1] años) se identificaron 554 casos incidentes de hipertensión entre 43.562 personasaño. La hazard ratio (HR) de hipertensión para un consumo de alcohol $\geq 5$ días por semana fue 1,28 (intervalo de confianza $[\mathrm{IC}]$ del 95\%, 0,97-1,70) comparados con abstemios. Entre consumidores de alcohol al menos 5 días por semana, la HR de hipertensión para consumos $\geq 1$ copa al día fue 1,45 (IC del 95\%, 1,06-2) comparados con los abstemios. El consumo de cerveza y licores, pero no de vino, se asoció con mayor riesgo de hipertensión. La HR para el consumo diario de más de 0,5 copas de cerveza y licores, en comparación con los no bebedores, fue 1,53 (IC del 95\%, 1,18-1,99). En cambio, la asociación entre el consumo de vino tinto y el riesgo de hipertensión fue inversa pero no significativa.

Conclusiones. En esta población mediterránea, el consumo de cerveza y licores, pero no de vino, se asoció con mayor riesgo de hipertensión. El patrón de consumo semanal de alcohol, sin embargo, no tuvo un impacto significativo en el riesgo de hipertensión.

Palabras clave: Hipertensión. Vino tinto. Patrón de consumo de alcohol. Estudios prospectivos. Dieta mediterránea.

\section{VÉASE EDITORIAL EN PÁGS. 603-5}

Este proyecto ha sido financiado por el Ministerio de Sanidad y Consumo (FIS: PI030678, PI040233, PI042241, PI050514, PI050976 PI070240 y RD06/0045) y el Gobierno de Navarra (PI41/2005).

Correspondencia: Dr. J.M. Núñez-Córdoba.

Departamento de Medicina Preventiva y Salud Pública. Clínica

Universitaria de Navarra. Universidad de Navarra.

Irunlarrea, 1. 31008 Pampona. Navarra. España.

Correo electrónico: jorge.nun@gmail.com

Recibido el 9 de julio de 2008.

Aceptado para su publicación el 19 de febrero de 2009.

\section{Alcohol Consumption and the Incidence of Hypertension in a Mediterranean Cohort: the SUN Study}

Introduction and objectives. To assess prospectively the association between alcohol consumption, including alcoholic beverage preference and days of consumption per week, and the risk of hypertension in a Mediterranean cohort.

Methods. We prospectively followed 9,963 Spanish men and women initially without hypertension. Self-reported and validated data on diet and hypertension diagnoses were collected.

Results. During follow-up (median [interquartile range], 4.2 [2.5-6.1] years), 554 incident cases of hypertension were identified over a total of 43,562 person-years. The hazard ratio for hypertension among those who consumed alcohol on $\geq 5$ days per week was 1.28 (95\% confidence interval, 0.97-1.7) compared to abstainers. Among those who drank alcohol $\geq 5$ days per week, the hazard ratio for hypertension associated with consuming $\geq 1$ drink per day was 1.45 (95\% confidence interval, 1.06-2) compared with abstainers. The consumption of beer or spirits, but not wine, was associated with an increased risk of hypertension. The hazard ratio associated with consuming $>0.5$ drinks of beer or spirits per day was 1.53 (95\% confidence interval, 1.18-1.99) compared with abstainers. In contrast, there was a nonsignificant inverse association between red wine intake and the risk of hypertension.

Conclusions. In this Mediterranean population, the consumption of beer or spirits, but not wine, was associated with a higher risk of developing hypertension. However, the weekly pattern of alcohol consumption did not have a significant impact on the risk of hypertension.

Key words: Hypertension. Red wine. Alcohol consumption pattern. Prospective studies. Mediterranean diet.

Full English text available from: www.revespcardiol.org

\section{INTRODUCCIÓN}

Siguen sin resolverse algunas cuestiones referentes al balance entre los riesgos para la salud y los beneficios cardiovasculares que presenta el consumo de alcohol. Aunque el consumo moderado de 


\section{ABREVIATURAS}

AGMI: ácidos grasos monoinsaturados.

AGPI: ácidos grasos poliinsaturados.

AGS: ácidos grasos saturados.

IMC: índice de masa corporal.

MET: equivalente metabólico.

RIC: intervalo intercuartílico.

alcohol muestra un efecto protector contra varias enfermedades cardiovasculares ${ }^{1-6}$, el consumo de alcohol en exceso es uno de los principales factores de riesgo de hipertensión, la segunda causa más importante de pérdida de años de vida ajustados por discapacidad en el mundo ${ }^{7}$ y una enfermedad con alta prevalencia que contribuye a importantes efectos adversos para la salud, como muerte prematura, morbilidad coronaria, insuficiencia renal y accidentes cerebrovasculares.

El efecto de la cantidad de alcohol en relación con la hipertensión y de acuerdo con la frecuencia de bebida aún no se ha aclarado suficientemente y la mayoría de los estudios que examinan la relación entre el consumo de alcohol y la hipertensión tienen un diseño transversal. Los datos epidemiológicos de estudios prospectivos son escasos y los resultados no concuerdan. Se ha señalado que el riesgo de hipertensión asociado a un consumo elevado de alcohol es independiente del tipo específico de bebida alcohólica consumida ${ }^{8}$. Sin embargo, en un reciente estudio de cohortes, se observaron elevaciones significativas del riesgo de hipertensión solamente en las categorías más altas de consumo de cerveza, licor y vino blanco, pero no de vino tinto ${ }^{9}$. Además, la influencia del patrón de consumo de alcohol, incluyendo los tipos específicos de bebida, en el riesgo de hipertensión arterial no se ha estudiado de modo prospectivo en una población mediterránea, en la que a priori el mayor consumo de vino y la consecuentemente mayor variación de dicho consumo entre sujetos permitiría valorar mejor su efecto.

Se evaluó la relación entre el tipo, la cantidad y la frecuencia de bebida alcohólica y la incidencia de hipertensión en el estudio Seguimiento Universidad de Navarra (SUN), una cohorte prospectiva de graduados universitarios en España.

\section{MÉTODOS}

\section{Población de estudio}

El estudio SUN es una cohorte dinámica prospectiva con un reclutamiento permanentemente abierto, cuyos participantes son todos graduados universitarios, contactados y seguidos mediante cuestionarios enviados por correo. Los métodos del estudio se han descrito con más detalle en una publicación previa ${ }^{10}$. Brevemente, desde diciembre de 1999, se envían por correo cuestionarios junto con la carta de invitación a participar en el estudio SUN a todos los graduados de la Universidad de Navarra, profesionales de enfermería registrados de algunas provincias españolas y graduados universitarios de otros colegios y asociaciones profesionales. Responder al cuestionario inicial se consideró consentimiento informado para participar en el estudio. El protocolo del proyecto fue aprobado por el Comité de Ética de la Universidad de Navarra.

Después de la evaluación basal, cada 2 años los participantes recibieron por correo cuestionarios de seguimiento, donde se recoge una amplia variedad de información acerca de dieta, estilos de vida, factores de riesgo y enfermedades. A quienes no responden a los cuestionarios de seguimiento, se les envían hasta cinco correos de recordatorio para alcanzar una retención óptima.

En febrero de 2008, el estudio SUN había reclutado a 19.057 participantes, con edades basales comprendidas entre los 20 y los 90 años. Entre ellos, se reclutó a 15.552 individuos antes de mayo de 2005. Se excluyó a 1.739 participantes con hipertensión basal, 1.544 con antecedente de enfermedad cardiovascular, cáncer o diabetes mellitus basales, 1.563 con ingesta energética total y basal por encima o por debajo de los límites preestablecidos (ingesta energética total diaria $<500 \mathrm{o}>3.500 \mathrm{kcal}$ las mujeres y $<800$ o $>4.000 \mathrm{kcal}$ los varones), y 332 con valores de otras covariables perdidos. Finalmente, 11.279 participantes quedaron disponibles para el estudio, de los que 9.963 respondieron, al menos, un cuestionario de seguimiento.

\section{Evaluación dietética}

Los hábitos dietéticos basales se evaluaron mediante un cuestionario semicuantitativo de frecuencia de consumo de alimentos con 136 ítems previamente validado en España ${ }^{11}$. El cuestionario se basa en tamaños de raciones típicos y cuenta con nueve opciones de frecuencia de consumo para cada alimento (desde nunca o casi nunca $a \geq 6$ veces al día) durante el año anterior. El consumo de bebidas alcohólicas se evaluó en cinco de esos ítems y hubo seis adicionales referentes al patrón de consumo de alcohol. En uno se incluyó la siguiente pregunta: «Por término medio en una semana típica, ¿cuántos días/semana bebes alcohol (vino, cerveza o licores destilados), incluyendo el que tomas en las comidas?». Un equipo de dietistas cualificadas actualizó la base de datos de nutrientes de acuerdo con la última información disponible utilizando las tablas 
de composición de alimentos para España ${ }^{12,13}$. Las personas que no consumían alcohol se definieron como no bebedores actualmente. La preferencia de bebida (vino o cerveza y licores) se estableció atendiendo al tipo de bebida del que procediera al menos el 50\% del consumo total de alcohol de cada participante.

\section{Evaluación de otras covariables}

El cuestionario basal recogió información sobre factores sociodemográficos (sexo, edad, grado universitario, estado civil, situación laboral), medidas antropométricas (peso, talla), hábitos relacionados con la salud (consumo de tabaco, actividad física), y variables clínicas (medicación actual, historia familiar y personal de hipertensión, enfermedad coronaria, cáncer y otras enfermedades). El índice de masa corporal (IMC) se definió como el peso (en kilogramos) dividido por el cuadrado de la estatura (en metros). El cuestionario evaluó la participación y el tiempo empleado en 17 actividades diferentes. Se asignó un múltiplo de la puntuación de equivalente metabólico (MET) para cada una de estas actividades, usando las guías publicadas previamente para cuantificar la intensidad media de la actividad física $^{14}$. Los estudios sobre la validez del peso, el IMC y la actividad física en el tiempo libre declarados por los sujetos en el estudio SUN han sido publicados previamente ${ }^{15,16}$.

\section{Evaluación de la hipertensión}

En el cuestionario basal y en los de seguimiento, se preguntó a los participantes si tenían diagnóstico médico de hipertensión y la fecha de éste. En el cuestionario basal también se recogió información sobre los valores más recientes de presión sistólica y diastólica. En el cuestionario de seguimiento se preguntó a los participantes si se habían medido la presión arterial desde que completaron el cuestionario previo.

Se consideró hipertensos en la evaluación basal a los individuos que presentaron un diagnóstico médico de hipertenión, presión arterial sistólica $\geq 140$ $\mathrm{mmHg}$ o presión arterial diastólica $\geq 90 \mathrm{mmHg}$ o tomaban medicación antihipertensiva ${ }^{17}$. Los casos incidentes de hipertensión fueron los participantes que no presentaban hipertensión en la evaluación basal y recibieron un diagnóstico médico de hipertensión en el cuestionario de seguimiento. El diagnóstico de hipertensión en esta cohorte se ha validado en un estudio previo ${ }^{18}$. Entre los participantes que presentaron diagnóstico de hipertensión, el 82,3\% (intervalo de confianza [IC] del 95\%, 72,8-92,8) de los casos fueron confirmados en una visita domiciliaria en la que un médico midió dos veces la presión arterial de los participantes, con enmascaramiento de la información declarada en el cuestionario, empleando un protocolo estandarizado. Entre los participantes que no tenían diagnóstico médico de hipertensión, el 85,4\% (IC del 95\%, $72,4-89,1)$ fueron confirmados como no hipertensos durante la visita domiciliaria ${ }^{18}$.

\section{Análisis estadístico}

Se estimaron las hazard ratio (HR) de hipertensión y sus respectivos IC del 95\% a través de las categorías de consumo de alcohol empleando modelos de riesgos proporcionales (regresión de Cox).

De acuerdo con la actual recomendación referente al tratamiento de los posibles factores de confusión ${ }^{19}$, se tuvo en cuenta la literatura científica previamente publicada sobre los factores de riesgo causales de hipertensión, evitando basarse en aproximaciones exclusivamente estadísticas tales como los criterios basados en los valores de $\mathrm{p}$, los procedimientos paso a paso (stepwise) o el simple cambio del valor de la estimación puntual al ajustar por cada variable candidata a ser factor de confusión, estrategias de las que se ha demostrado que pueden conducir a errores ${ }^{19}$. Además de ajustar por esos factores de riesgo causales, se ajustó también por variables fuertemente asociadas al estilo de vida, como el consumo de tabaco, porque su control puede ayudar a controlar la confusión.

De este modo, se ajustó por los siguientes factores basales: edad (variable continua), sexo, consumo energético total (quintiles), IMC (continua), actividad física (quintiles), antecedentes familiares de hipertensión (sí/no), hipercolesterolemia (sí/ no), ingesta de sodio (quintiles), ingesta de potasio (quintiles), consumo de lácteos pobres en grasa (quintiles), consumo de frutas (quintiles), consumo de verduras y hortalizas (quintiles), consumo de aceite de oliva (quintiles), consumo de fibra cereal (quintiles), consumo de proteínas de origen vegetal (quintiles), consumo de cafeína (quintiles), consumo de pescado (quintiles) y consumo de tabaco (nunca fumador, ex fumador o fumador actual). El supuesto de proporcionalidad se verificó mediante la introducción en el modelo de Cox de una interacción entre la variable explicativa y el tiempo.

La ingesta de alimentos y nutrientes se ajustó por la ingesta energética total mediante el método de los residuales, empleándose modelos de regresión separados para obtener los residuales de acuerdo con el sexo ${ }^{20}$. La copa estándar de bebida alcohólica se definió como $13,7 \mathrm{~g}$ de alcohol puro $^{21}$. Salvo en los casos indicados, se consideró al grupo de los no bebedores como la categoría de referencia en la mayoría de los análisis. 
TABLA 1. Características basales de acuerdo con el consumo de copas de alcohol y preferencia de bebida alcohólica. Estudio SUN, 1999-2005

\begin{tabular}{|c|c|c|c|c|c|c|}
\hline & \multicolumn{3}{|c|}{ Consumo de copas/día } & \multicolumn{3}{|c|}{ Preferencia de bebida alcohólica } \\
\hline & $\begin{array}{c}0 \\
(n=2.190)\end{array}$ & $\begin{array}{c}0,1-0,5 \\
(n=4.706)\end{array}$ & $\begin{aligned} & >0,5 \\
(n & =3.067)\end{aligned}$ & $\begin{array}{c}\text { Vino } \\
(n=2.751)\end{array}$ & $\begin{array}{l}\text { Cerveza y licores } \\
\quad(n=5.022)\end{array}$ & $\begin{array}{l}\text { No bebedores } \\
(n=2.190)\end{array}$ \\
\hline Edad (años) & $35,5 \pm 10,7$ & $35,1 \pm 10,3$ & $38,5 \pm 10,8$ & $40,2 \pm 10,5$ & $34,4 \pm 10,2$ & $35,5 \pm 10,7$ \\
\hline Mujeres, \% & 80,9 & 68,3 & 39,3 & 55,2 & 57,8 & 80,9 \\
\hline IMC & $22,6 \pm 3,1$ & $22,9 \pm 3,1$ & $24 \pm 3,2$ & $23,6 \pm 3,2$ & $23,1 \pm 3,2$ & $22,6 \pm 3,1$ \\
\hline Actividad física (MET-h/semana) & $23,6 \pm 24,4$ & $23,4 \pm 19,8$ & $26,5 \pm 22$ & $24,4 \pm 20,2$ & $24,8 \pm 21,1$ & $23,6 \pm 24,4$ \\
\hline Hipercolesterolemia, \% & 12,8 & 11,8 & 17,4 & 17,2 & 12,3 & 12,8 \\
\hline Antecedentes familiares de hipertensión, \% & 48,1 & 48,8 & 48,6 & 52,2 & 46,8 & 48,1 \\
\hline Fumadores, $\%$ & 16 & 23,9 & 29,2 & 19,7 & 29,4 & 16 \\
\hline Consumo de alcohol (g/día) & 0 & $2,7 \pm 1,7$ & $16 \pm 11,5$ & $9,2 \pm 11,3$ & $7,2 \pm 8,8$ & 0 \\
\hline \multicolumn{7}{|l|}{ Preferencia de bebida alcohólica, \% } \\
\hline Vino & 0 & 33 & 39,1 & & & \\
\hline Ceveza y licores & 0 & 67 & 60,9 & & & \\
\hline \multicolumn{7}{|l|}{ Ingesta diaria } \\
\hline Sodio (g) & $3,2 \pm 2,1$ & $3,3 \pm 2,2$ & $3,6 \pm 2,2$ & $3,4 \pm 2,3$ & $3,4 \pm 2,1$ & $3,2 \pm 2,1$ \\
\hline Potasio (g) & $4,7 \pm 1,6$ & $4,7 \pm 1,5$ & $4,7 \pm 1,4$ & $4,8 \pm 1,5$ & $4,6 \pm 1,5$ & $4,7 \pm 1,6$ \\
\hline Calcio $(\mathrm{g})$ & $1,3 \pm 0,5$ & $1,2 \pm 0,5$ & $1,2 \pm 0,4$ & $1,2 \pm 0,5$ & $1,2 \pm 0,5$ & $1,3 \pm 0,5$ \\
\hline Magnesio (mg) & $406 \pm 127$ & $408 \pm 118$ & $416 \pm 114$ & $418 \pm 122$ & $407 \pm 114$ & $406 \pm 127$ \\
\hline Frutas (raciones) & $2,9 \pm 2,2$ & $2,7 \pm 2,1$ & $2,5 \pm 1,9$ & $2,8 \pm 2,1$ & $2,6 \pm 2$ & $2,9 \pm 2,2$ \\
\hline Verduras (raciones) & $2,5 \pm 1,5$ & $2,6 \pm 1,4$ & $2,5 \pm 1,4$ & $2,6 \pm 1,5$ & $2,5 \pm 1,4$ & $2,5 \pm 1,5$ \\
\hline Fibra (g) & $27,1 \pm 12,6$ & $26,9 \pm 11,8$ & $25,9 \pm 10,8$ & $27,6 \pm 12$ & $25,9 \pm 11$ & $27,1 \pm 12,6$ \\
\hline Fibra de cereales (g) & $3,3 \pm 3,1$ & $3,4 \pm 2,9$ & $3,4 \pm 2,8$ & $3,5 \pm 3,1$ & $3,3 \pm 2,7$ & $3,3 \pm 3,1$ \\
\hline Cafeína (mg) & $37,5 \pm 41$ & $44,2 \pm 38,6$ & $51,8 \pm 39,6$ & $46,7 \pm 39,1$ & $47,5 \pm 39,3$ & $37,5 \pm 41$ \\
\hline Aceite de oliva (g) & $18,2 \pm 16$ & $18,5 \pm 14,7$ & $18,8 \pm 15$ & $19,2 \pm 15,6$ & $18,3 \pm 14,4$ & $18,2 \pm 16$ \\
\hline Productos lácteos pobres en grasa $(\mathrm{g})$ & $239,4 \pm 271,1$ & $225,8 \pm 250,2$ & $182,1 \pm 220,7$ & $212 \pm 239$ & $207 \pm 240$ & $239,4 \pm 271,1$ \\
\hline Pescado $(\mathrm{g})$ & $92,3 \pm 62,8$ & $93,2 \pm 56,8$ & $98,9 \pm 55,3$ & $99,5 \pm 59,3$ & $93,2 \pm 54,4$ & $92,3 \pm 62,8$ \\
\hline Ingesta energética total (kcal) & $2.308 \pm 622$ & $2.337 \pm 592$ & $2.485 \pm 616$ & $2.389 \pm 616$ & $2.399 \pm 600$ & $2.308 \pm 622$ \\
\hline \multicolumn{7}{|l|}{ Ingesta energética, \% } \\
\hline Hidratos de carbono & $44,6 \pm 7,7$ & $43,7 \pm 7,1$ & $41,9 \pm 7,1$ & $43,1 \pm 7,5$ & $42,9 \pm 6,9$ & $44,6 \pm 7,7$ \\
\hline Proteínas & $18,4 \pm 3,6$ & $18,2 \pm 3,2$ & $17,3 \pm 2,9$ & $17,9 \pm 3,2$ & $17,8 \pm 3$ & $18,4 \pm 3,6$ \\
\hline Proteínas de origen vegetal & $5,4 \pm 1,5$ & $5,3 \pm 1,2$ & $5,1 \pm 1,2$ & $5,3 \pm 1,2$ & $5,2 \pm 1,2$ & $5,4 \pm 1,5$ \\
\hline Lípidos & $37 \pm 7$ & $37,3 \pm 6,4$ & $36,1 \pm 6,2$ & $36,3 \pm 6,5$ & $37,1 \pm 6,2$ & $37 \pm 7$ \\
\hline AGS & $12,8 \pm 3,5$ & $12,8 \pm 3,1$ & $12,4 \pm 3$ & $12,4 \pm 3,1$ & $12,8 \pm 3$ & $12,8 \pm 3,5$ \\
\hline AGMI & $15,7 \pm 4$ & $16 \pm 3,7$ & $15,6 \pm 3,6$ & $15,7 \pm 3,8$ & $15,8 \pm 3,6$ & $15,7 \pm 4$ \\
\hline AGPI & $5,3 \pm 1,8$ & $5,3 \pm 1,6$ & $5,2 \pm 1,4$ & $5,2 \pm 1,5$ & $5,3 \pm 1,6$ & $5,3 \pm 1,8$ \\
\hline
\end{tabular}

Los valores expresan media \pm desviación estándar, salvo otra indicación.

Una copa estándar se definió como 13,7 g de alcohol puro.

La preferencia por bebida alcohólica de cada participante se definió de acuerdo con el tipo específico de bebida del que procediera al menos el $50 \%$ del alcohol total consumido por cada participante. Se consideró no bebedores a los participantes que refirieron no consumir ninguna bebida alcohólica.

Se realizaron tests de tendencia lineal a través de las categorías crecientes de días por semana de consumo de alcohol, tomando el valor mediano de cada categoría y tratando la variable resultante como una variable continua. La interacción estadística se evaluó mediante test de razón de verosimilitudes, comparando los modelos completos (incluyendo términos de interacción) con los modelos reducidos sin términos de interacción. Todos los valores de $\mathrm{p}$ se obtuvieron mediante pruebas bilaterales y la significación estadística se estableció en un valor de $\mathrm{p}<0,05$. Se empleó la versión 15.0 de SPSS (SPSS Inc., Chicago, Illinois, Estados Unidos) para todos los análisis.

\section{RESULTADOS}

\section{Características basales y consumo medio de alcohol}

Durante 43.562 persona-años de seguimiento, se identificaron 554 casos incidentes de hipertensión. La mediana [intervalo intercuartílico] de seguimiento fue 4,2 [2,5-6,1] años. Las características de los participantes de acuerdo con el consumo de alcohol y de la preferencia de bebida alcohólica se muestran en la tabla 1.

La mediana de copas consumidas al día fue 0,2 $[0,04-0,6]$. El número total de abstemios fue 2.190 
TABLA 2. Razón de riesgo de hipertensión de acuerdo con la frecuencia de consumo de alcohol y la cantidad basal de alcohol consumido entre los participantes del estudio SUN, 1999-2008

\begin{tabular}{|c|c|c|c|c|c|c|c|}
\hline \multicolumn{6}{|c|}{ Consumo semanal } & \multicolumn{2}{|r|}{ p (tendencia) } \\
\hline & $0^{\mathrm{a}}$ & \multicolumn{2}{|c|}{ 1-2 días } & \multicolumn{2}{|c|}{ 3-4 días } & $\geq \mathbf{5}$ días & \\
\hline Casos incidentes & 94 & \multicolumn{2}{|c|}{222} & \multicolumn{2}{|c|}{69} & 169 & \\
\hline Personas-año & 9.569 & \multicolumn{2}{|c|}{23.298} & \multicolumn{2}{|c|}{4.031} & 6.664 & \\
\hline HR (IC del 95\%) & 1 & \multicolumn{2}{|c|}{$0,94(0,74-1,20)$} & \multicolumn{2}{|c|}{$1,22(0,88-1,68)$} & $1,23(0,94-1,61)$ & 0,02 \\
\hline HR multivariable (IC del 95\%)c & 1 & \multicolumn{2}{|c|}{$0,97(0,76-1,25)$} & \multicolumn{2}{|c|}{$1,18(0,85-1,63)$} & $1,28(0,97-1,70)$ & 0,01 \\
\hline Consumo semanal (copas/día) & 0 & $<1$ & $\geq 1$ & $<1$ & $\geq 1$ & $<1$ & $\geq 1$ \\
\hline Casos incidentes & 94 & 210 & 12 & 51 & 18 & 74 & 95 \\
\hline Personas-año & 9.569 & 22.188 & 1.110 & 3.019 & 1.012 & 3.345 & 3.319 \\
\hline HR (IC del 95\%) & 1 & $0,95(0,74-1,21)$ & $0,89(0,49-1,64)$ & $1,21(0,85-1,71)$ & $1,26(0,75-2,11)$ & $1,06(0,77-1,46) \quad 1,4$ & ,40 (1,03-1,89) \\
\hline HR multivariable (IC del 95\%)c & 1 & $0,98(0,76-1,26)$ & $0,95(0,51-1,76)$ & $1,22(0,86-1,74)$ & $1,10(0,65-1,86)$ & $1,13(0,82-1,57) \quad 1,4$ & ,45 $(1,06-2,00)$ \\
\hline
\end{tabular}

HR: hazard ratio; IC: intervalo de confianza.

aCategoría de referencia.

${ }^{b}$ Ajustado por edad y sexo.

'Ajustado por edad y sexo, ingesta energética total, índice de masa corporal, actividad física, historia familiar de hipertensión, hipercolesterolemia, ingesta de sodio, ingesta de potasio, consumo de lácteos pobres en grasa, consumo de frutas, consumo de verduras, consumo de aceite de oliva, consumo de fibra cereal, consumo de proteína de origen vegetal, consumo de cafeína, consumo de pescado y consumo de tabaco.

$(22 \%)$, y entre ellos fue mayor la proporción de mujeres y no fumadores, mientras que quienes tenían mayor consumo de alcohol tenían una media de edad mayor, así como mayores consumos de cafeína y pescado y menor consumo de productos lácteos. En esta población, la preferencia mayoritaria de tipo de bebida alcohólica entre los bebedores fue la cerveza y los licores. Las demás características basales fueron similares entre las distintas categorías de consumo de alcohol.

El modelo multivariable mostró mayor riesgo de hipertensión entre los individuos con un consumo de más de 2 copas al día en comparación con los no bebedores $(\mathrm{HR}=1,55$; IC del 95\%, 1,04-2,32).

\section{Días de consumo de alcohol por semana, número de copas al día y riesgo de hipertensión}

Se evaluó la asociación de la frecuencia de consumo de alcohol y la cantidad de alcohol consumida por día de consumo con el riesgo de hipertensión arterial incidente (tabla 2).

En el análisis multivariable, un incremento del número de días de consumo se asoció débilmente con el riesgo de hipertensión aumentado. La HR de hipertensión entre quienes consumían alcohol al menos 5 días por semana fue 1,28 (IC del 95\%, 0,97-1,7) comparados con los no bebedores. Los participantes que bebieron más de 5 días por semana pero consumieron menos de 1 copa al día presentaron una HR ajustada de 1,13 (IC del 95\%, $0,82-1,57)$ comparados con los abstemios, mientras que esta HR fue de 1,45 (IC del 95\%, 1,06-2) para quienes consumieron al menos 1 copa al día y más de 5 días/semana.

Se evaluó también el riesgo de hipertensión de acuerdo con los días de consumo/semana a través de las diferentes categorías de consumo total de alcohol semanal (g/semana) (tabla 3). Entre los que presentaron el consumo más alto de alcohol (> $45 \mathrm{~g} / \mathrm{semana}$ ), un consumo de alcohol/semana $>2$ días se asoció con un riesgo de hipertensión elevado comparados con los que consumían alcohol 1-2 días/semana, aunque esta asociación no fue estadísticamente significativa $(\mathrm{HR}=1,33$; $\mathrm{IC}$ del 95\%, 0,96-1,83).

No se observó una asociación clara entre el número de días de consumo/semana y el riesgo de hipertensión entre los individuos con menor consumo semanal de alcohol total. No se observó ninguna interacción entre los días de consumo por semana y la edad (variable continua), el sexo o el IMC (continua).

\section{Preferencia de bebida alcohólica, tipo de bebida y riesgo de hipertensión}

Entre los bebedores, se evaluó si la preferencia de bebida alcohólica de acuerdo con el cuestionario basal se asociaba con el riesgo de hipertensión (tabla 4). Los individuos que mostraron preferencia por el consumo de cervezas y licores tenían un ligero aumento del riesgo de hipertensión comparados con los que tenían preferencia por consumo de vino, aunque la asociación no fue estadísticamente significativa $(\mathrm{HR}=1,18$; IC del 95\%, 0,97$1,44)$. 
TABLA 3. Razón de riesgo de hipertensión entre bebedores de acuerdo con la frecuencia de consumo de alcohol y la cantidad total de alcohol consumida por semana. Estudio SUN, 1999-2008

\begin{tabular}{|c|c|c|}
\hline \multirow[t]{2}{*}{ Ingesta total de alcohol } & \multicolumn{2}{|c|}{ Consumo semanal (días) } \\
\hline & $\leq 2^{\mathrm{a}}$ & $>2$ \\
\hline \multicolumn{3}{|l|}{$>0-30 \mathrm{~g} / \mathrm{semana}$} \\
\hline Casos incidentes & 127 & 33 \\
\hline Personas-año & 14.641 & 1.651 \\
\hline HR (IC del 95\%) ${ }^{b}$ & 1,00 & $1,16(0,77-1,74)$ \\
\hline HR multivariable 1 (IC del 95\%) ${ }^{c}$ & 1,00 & $1,19(0,78-1,81)$ \\
\hline HR multivariable 2 (IC del 95\%) ${ }^{d}$ & 1,00 & $1,22(0,80-1,87)$ \\
\hline \multicolumn{3}{|l|}{$>30-45 \mathrm{~g} /$ semana } \\
\hline Casos incidentes & 37 & 18 \\
\hline Personas-año & 3.016 & 1.036 \\
\hline HR (IC del 95\%) ${ }^{b}$ & 1 & $0,85(0,47-1,54)$ \\
\hline HR multivariable 1 (IC del 95\%) ${ }^{c}$ & 1 & $0,81(0,41-1,63)$ \\
\hline HR multivariable 2 (IC del 95\%) ${ }^{d}$ & 1 & $0,76(0,38-1,53)$ \\
\hline \multicolumn{3}{|l|}{$>45 \mathrm{~g} /$ semana } \\
\hline Casos incidentes & 58 & 187 \\
\hline Personas-año & 5.641 & 8.008 \\
\hline HR (IC del 95\%) ${ }^{b}$ & 1 & $1,40(1,03-1,90)$ \\
\hline HR multivariable 1 (IC del 95\%) ${ }^{c}$ & 1 & $1,40(1,03-1,91)$ \\
\hline HR multivariable 2 (IC del 95\%) ${ }^{d}$ & 1 & $1,33(0,96-1,83)$ \\
\hline
\end{tabular}

HR: hazard ratio; IC: intervalo de confianza.

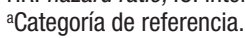

'DAjustado por edad y sexo.

'Ajustado por edad, sexo, ingesta energética total, IMC, actividad física, historia familiar de hipertensión, hipercolesterolemia, ingesta de sodio, ingesta de potasio consumo de lácteos pobres en grasa, consumo de frutas, consumo de verduras, consumo de aceite de oliva, consumo de fibra cereal, consumo de proteína de origen vegetal, consumo de cafeína, consumo de pescado y consumo de tabaco.

${ }^{\mathrm{d} A j u s t a d o}$ por las variables mencionadas más consumo de alcohol.

Se analizó también la asociación entre el consumo de vino tinto, el consumo de otros tipos de vino y el consumo de cerveza y licores con el riesgo de hipertensión (tabla 5). El consumo de cerveza y licores se asoció con un mayor riesgo de hipertensión ( $\mathrm{HR}=1,53$; IC del 95\%, 1,18-1,99) en aquellos que consumían más de 0,5 unidades de bebida/día respecto a los que no consumían cerveza y licores. Esta asociación no se observó en los que mostraban preferencia por el vino ni en los que mostraban preferencia por otros tipos de vino.

Atendiendo a la estimación puntual de la HR, se observó una relación inversa no significativa entre el consumo de vino tinto y el riesgo de hipertensión.

No se observó ninguna interacción entre el tipo de bebida consumida y la edad (variable continua), el sexo o el IMC (continua).

\section{DISCUSIÓN}

En esta cohorte prospectiva mediterránea, el consumo total de alcohol pero no el patrón de consumo (días de consumo/semana) se asoció de manera clara con el riesgo de hipertensión. Por otro lado, se observó que los individuos con preferencia por cerveza y licores tenían un riesgo de hipertensión ligeramente mayor que quienes mostraban preferencia por el vino. Además, el consumo de alcohol procedente de cerveza y licores, pero no de vino, se asoció con un riesgo de hipertensión incrementado.

Este trabajo es el primer estudio prospectivo a largo plazo que ha evaluado la asociación entre el consumo de alcohol (incluidos el patrón semanal de bebida y el tipo de bebida alcohólica) y la incidencia de hipertensión arterial en una cohorte mediterránea. Como cabía esperar, los resultados concuerdan con la hipótesis que establece que un consumo excesivo de alcohol incrementa el riesgo de hipertensión arterial ${ }^{22-24}$ y respalda las recomendaciones generales sobre la reducción del consumo de alcohol como medida para prevenir la hipertensión arterial ${ }^{25}$.

Se ha señalado que los efectos en la salud del consumo de alcohol pueden depender del patrón de consumo ${ }^{26}$. Los resultados obtenidos en nuestro estudio no concuerdan con esa hipótesis. Sin embargo, los hallazgos concuerdan con las conclusiones de un trabajo que estudió la asociación del consumo de alcohol y el patrón de bebida en una muestra de la población de Estados Unidos, que indicó que la media del alcohol total consumido

TABLA 4. Razón de riesgo de hipertensión de acuerdo con la preferencia de bebida alcohólica tomando como referencia a los participantes no bebedores. Estudio SUN, 1999-2008

\begin{tabular}{lccc}
\hline & No bebedores ${ }^{\mathbf{~}(\mathbf{n}=\mathbf{2 . 1 9 0})}$ & Vino $(\mathbf{n}=\mathbf{2 . 7 5 1 )}$ & Cerveza y licores $(\mathbf{n}=\mathbf{5 . 0 2 2})$ \\
\hline Casos incidentes & 94 & 189 & 271 \\
Personas-año & 9.569 & 12.032 & 21.961 \\
HR (IC del 95\%) & 1 & $1(0,78-1,29)$ & $1(0,85-1,38)$ \\
HR multivariable (IC del 95\%) & 1 & $0,99(0,77-1,29)$ & $1,13(0,88-1,45)$ \\
\hline
\end{tabular}

HR: hazard ratio; IC: intervalo de confianza.

aCategoría de referencia.

${ }^{\mathrm{b}}$ Ajustado por edad y sexo.

${ }^{c} A j u s t a d o$ por edad, sexo, ingesta energética total, IMC, actividad física, historia familiar de hipertensión, hipercolesterolemia, ingesta de sodio, ingesta de potasio, consumo de lácteos pobres en grasa, consumo de frutas, consumo de verduras, consumo de aceite de oliva, consumo de fibra cereal, consumo de proteína de origen vegetal, consumo de cafeína, consumo de pescado y consumo de tabaco. 
TABLA 5. Razón de riesgo de hipertensión de acuerdo con el tipo de bebida alcohólica. Estudio SUN, 1999-2008

\begin{tabular}{|c|c|c|c|c|c|}
\hline & Copas/día & Casos incidentes & HR (IC del 95\%) & HR multivariable 1 (IC del 95\%) & HR multivariable 2 (IC del 95\%) \\
\hline \multirow[t]{3}{*}{ Vino tinto } & $0^{d}$ & 216 & 1 & 1 & 1 \\
\hline & $0,1-0,5$ & 233 & $1,04(0,86-1,25)$ & $1,01(0,84-1,23)$ & $1,00(0,82-1,21)$ \\
\hline & $>0,5$ & 105 & $0,98(0,77-1,25)$ & $0,94(0,73-1,21)$ & $0,89(0,69-1,15)$ \\
\hline p (tendencia) & & & 0,79 & 0,59 & 0,33 \\
\hline \multirow[t]{3}{*}{ Otros tipos de vino } & $0^{d}$ & 383 & 1 & 1 & 1 \\
\hline & $0,1-0,5$ & 152 & $1,03(0,85-1,25)$ & $0,98(0,81-1,19)$ & $0,96(0,79-1,17)$ \\
\hline & $>0,5$ & 19 & $1,69(1,06-2,68)$ & $1,36(0,84-2,21)$ & $1,27(0,78-2,07)$ \\
\hline $\mathrm{p}$ (tendencia) & & & 0,03 & 0,22 & 0,35 \\
\hline \multirow[t]{3}{*}{ Cervezas y licores } & $0^{\mathrm{d}}$ & 148 & 1 & 1 & 1 \\
\hline & $0,1-0,5$ & 259 & $1,01(0,82-1,24)$ & $1,04(0,84-1,28)$ & $1,04(0,84-1,28)$ \\
\hline & $>0,5$ & 147 & $1,49(1,17-1,90)$ & $1,54(1,19-1,99)$ & $1,53(1,18-1,99)$ \\
\hline $\mathrm{p}$ (tendencia) & & & $<0,001$ & $<0,001$ & $<0,001$ \\
\hline
\end{tabular}

HR: hazard ratio; IC: intervalo de confianza.

aAjustado por edad y sexo.

${ }^{b}$ Ajustado por edad, sexo, ingesta energética total, IMC, actividad física, historia familiar de hipertensión, hipercolesterolemia, ingesta de sodio, ingesta de potasio, consumo de lácteos pobres en grasa, consumo de frutas, consumo de verduras, consumo de aceite de oliva, consumo de fibra cereal, consumo de proteína de origen vegetal, consumo de cafeína, consumo de pescado y consumo de tabaco.

${ }^{c}$ Ajustado por las variables mencionadas y por el consumo de alcohol procedente de las demás fuentes.

¿Categoría de referencia.

podría tener un papel más importante en la relación entre el consumo de alcohol y el riesgo de hipertensión que la frecuencia de consumo ${ }^{27}$.

Los resultados de nuestro estudio indican un riesgo de hipertensión entre los bebedores de vino tinto ligeramente menor que en los no bebedores, aunque los intervalos de confianza incluían el valor nulo. Este hallazgo concuerda con la conocida teoría a favor del efecto beneficioso del vino, la denominada paradoja francesa ${ }^{28,29}$. Sin embargo, este beneficio potencial del vino tinto contra la hipertensión ha sido cuestionado, por ejemplo, en un ensayo controlado y aleatorizado con 28 varones no abstemios que mostraron un incremento de la presión arterial causado por un consumo moderado de alcohol independientemente de la fuente de procedencia de éste ${ }^{30}$. Sin embargo, este ensayo a corto plazo no es directamente comparable con la evaluación del efecto a largo plazo mediante estudios de grandes cohortes. Por otro lado, hay pruebas acerca de algunos de los mecanismos de los potenciales efectos beneficiosos del vino, como los efectos de los polifenoles encontrados en el vino tinto, especialmente el resveratrol, que ha mostrado una inhibición de la angiotensina II y un incremento de la síntesis de oxido nítrico, lo que explicaría parcialmente la reducción de presión arterial observada en algunos modelos renales de hipertensión ${ }^{31}$.

Los efectos beneficiosos del vino en comparación con otras bebidas alcohólicas podrían explicarse por factores de confusión relacionados con los hábitos dietéticos u otros estilos de vida ${ }^{32}$. Sin embargo, en nuestra cohorte se ha constatado un patrón dietético similar entre los bebedores de vino tinto y los bebedores de otros tipos de bebida ${ }^{33}$. Además, en los análisis realizados se controló por potenciales factores de confusión tanto dietéticos como relacionados con otros aspectos de los estilos de vida.

Nuestros resultados sostienen indirectamente la hipótesis de que hay un efecto beneficioso del vino en comparación con otras bebidas alcohólicas respecto a la prevención de la enfermedad cardiovascular y la mortalidad ${ }^{34}$. Acorde con nuestros resultados, en un reciente estudio de cohortes se ha observado un incremento significativo del riesgo de hipertensión sólo con el consumo de cerveza, licores y vino blanco, mientras que el consumo de vino tinto no mostró ningún efecto significativo?.

Nuestro estudio tiene varias limitaciones. Podría darse un sesgo de mala clasificación respecto a la exposición dietética a pesar de la buena correlación entre los cuestionarios de frecuencia alimentaria y la dieta habitual ${ }^{35}$. Sin embargo, el estudio de validación del cuestionario empleado en nuestro estudio demostró validez y fiabilidad adecuadas ${ }^{11}$. Además, el alcohol fue el nutriente que mostró la más alta correlación respecto al registro dietético en el estudio de validación del cuestionario de frecuencia de consumo de alimentos empleado en nuestro estudio ${ }^{11}$. Podría pensarse en una inversión de la secuencia temporal entre causa y efecto como explicación alternativa a los resultados. Sin embargo, el carácter prospectivo del estudio SUN reduce en gran medida la probabilidad de tal sesgo de causalidad inversa. Además, se excluyó del seguimiento a los individuos con diagnóstico basal de hipertensión o enfermedad cardiovascular prevalente en la evaluación basal.

Más allá de estas limitaciones se encuentra el hecho de que los diagnósticos de hipertensión 
fueran declarados por los sujetos. Sin embargo, la validez de la medida del diagnóstico de hipertensión ha sido demostrada en un estudio previo ${ }^{18}$.

Por último, nuestros hallazgos deberían interpretarse con cierta precaución porque no se puede garantizar que la confusión residual haya sido eliminada totalmente, a pesar de que en los análisis realizados se ajustó por los principales factores de riesgo de hipertensión y varios factores dietéticos. Futuros estudios que utilicen muestras representativas de la población general, cuyos estilo de vida y hábitos dietéticos podrían ser diferentes y en la que probablemente habría mayor variabilidad en el consumo de alcohol, estarían justificados para poder confirmar las conclusiones del nuestro.

\section{CONCLUSIONES}

Estos resultados indican un incremento del riesgo de hipertensión arterial asociado al consumo de alcohol, sin ningún papel específico del patrón de bebida. Por otra parte, sólo la cerveza y los licores, pero no el vino tinto, mostraron un efecto perjudicial en cuanto al riesgo de contraer hipertensión.

\section{AGRADECIMIENTOS}

Agradecemos a los participantes del estudio SUN su colaboración constante, y a todos los miembros del equipo investigador del estudio SUN su valioso apoyo: J. de Irala, M. Segui-Gómez, F. Guillén-Grima, A. SánchezVillegas, C. Fuente, M. Delgado-Rodríguez, M. SerranoMartínez, Z. Vázquez, S. Benito y J. Krafka.

\section{BIBLIOGRAFÍA}

1. Rimm EB, Giovannucci EL, Willett WC, Colditz GA, Ascherio A, Rosner B, et al. Prospective study of alcohol consumption and risk of coronary disease in men. Lancet. 1991;338:464-8.

2. Klatsky AL. Moderate drinking and reduced risk of heart disease. Alcohol Res Health. 1999;23:15-23.

3. Hines LM, Rimm EB. Moderate alcohol consumption and coronary heart disease: a review. Postgrad Med J. 2001;77:747-52.

4. Mukamal KJ, Conigrave KM, Mittleman MA, Camargo CA $\mathrm{Jr}$, Stampfer MJ, Willett WC, et al. Roles of drinking pattern and type of alcohol consumed in coronary heart disease in men. N Engl J Med. 2003;348:109-18.

5. Mukamal KJ, Ascherio A, Mittleman MA, Conigrave KM, Camargo CA Jr, Kawachi I, et al. Alcohol and risk for ischemic stroke in men: the role of drinking patterns and usual beverage. Ann Intern Med. 2005;142:11-9.

6. Athyros VG, Liberopoulos EN, Mikhailidis DP, Papageorgiou AA, Ganotakis ES, Tziomalos K, et al. Association of drinking pattern and alcohol beverage type with the prevalence of metabolic syndrome, diabetes, coronary heart disease, stroke, and peripheral arterial disease in a Mediterranean cohort. Angiology. 2008;58:689-97.

7. López AD, Mathers CD, Ezzati M, Jamison DT, Murray CJ. Global and regional burden of disease and risk factors, 2001: systematic analysis of population health data. Lancet. 2006;367:1747-57.
8. Thadhani R, Camargo CA Jr, Stampfer MJ, Curhan GC, Willett WC, Rimm EB. Prospective study of moderate alcohol consumption and risk of hypertension in young women. Arch Intern Med. 2002;162:569-74.

9. Sesso HD, Cook NR, Buring JE, Manson JE, Gaziano JM. Alcohol consumption and the risk of hypertension in women and men. Hypertension. 2008;51:1080-7.

10. Seguí-Gómez M, De la Fuente C, Vázquez Z, De Irala J, Martínez-González MA. Cohort profile: the "Seguimiento Universidad de Navarra" (SUN) study. Int J Epidemiol. 2006:35:1417-22.

11. Martín-Moreno JM, Boyle P, Gorgojo L, Maisonneuve P, Fernandez-Rodriguez JC, Salvini S, et al. Development and validation of a food frequency questionnaire in Spain. Int J Epidemiol. 1993;22:512-9.

12. Mataix Verdú J. Tabla de composición de alimentos españoles. 4. ${ }^{a}$ ed. Granada: Universidad de Granada; 2003

13. Moreiras O, Carbajal A, Cabrera L, Cuadrado C. Tablas de composición de alimentos. 9. a ed. Madrid: Pirámide; 2005.

14. Ainsworth BE, Haskell WL, Whitt MC, Irwin ML, Swartz AM, Strath SJ, et al. Compendium of physical activities: an update of activity codes and MET intensities. Med Sci Sports Exerc. 2000;32:S498-504

15. Bes-Rastrollo M, Pérez Valdivieso JR, Sánchez-Villegas A, Alonso A, Martínez-González MA. Validación del peso e índice de masa corporal auto-declarados de los participantes de una cohorte de graduados universitarios. Rev Esp Obes. 2005;3:183-9.

16. Martínez-González MA, López-Fontana C, Varo JJ, SánchezVillegas A, Martínez JA. Validation of the Spanish version of the physical activity questionnaire used in the Nurses' Health Study and the Health Professionals' Follow-up Study. Public Health Nutr. 2005;8:920-7.

17. Chobanian AV, Bakris GL, Black HR, Cushman WC, Green LA, Izzo JL Jr, et al. The Seventh Report of the Joint National Committee on Prevention, Detection, Evaluation, and Treatment of High Blood Pressure: the JNC 7 report. JAMA. 2003;289:2560-72

18. Alonso A, Beunza JJ, Delgado-Rodríguez M, MartínezGonzález MA. Validation of self reported diagnosis of hypertension in a cohort of university graduates in Spain. BMC Public Health. 2005;5:94.

19. Hernan MA, Hernandez-Diaz S, Werler MM, Mitchell AA. Causal knowledge as a prerequisite for confounding evaluation: an application to birth defects epidemiology. Am J Epidemiol. 2002:155:176-84.

20. Willett W. Nutritional epidemiology. 2. ${ }^{a}$ ed. New York: Oxford University Press; 1998.

21. Centers for Disease Control and Prevention. General Information on Alcohol Use and Health [CDC Alcohol web site]. 2008 Ene. Disponible en: http:// www.cdc.gov/alcohol/ quickstats/general_info.htm

22. Ascherio A, Hennekens C, Willett WC, Sacks F, Rosner B, Manson J, et al. Prospective study of nutritional factors, blood pressure, and hypertension among US women. Hypertension. 1996;27:1065-72.

23. Tsuruta $M$, Adachi $H$, Hirai $Y$, Fujiura $Y$, Imaizumi $T$. Association between alcohol intake and development of hypertension in Japanese normotensive men: 12-year followup study. Am J Hypertens. 2000;13:482-7.

24. Fuchs FD, Chambless LE, Whelton PK, Nieto FJ, Heiss G. Alcohol consumption and the incidence of hypertension: The Atherosclerosis Risk in Communities Study. Hypertension. 2001;37:1242-50

25. Touyz RM, Campbell N, Logan A, Gledhill N, Petrella R, Padwal R, et al. The 2004 Canadian recommendations for the management of hypertension: Part III — Lifestyle modifications to prevent and control hypertension. Can J Cardiol. 2004;20:55-9. 
26. Seppa K, Laippala P, Sillanaukee P. Drinking pattern and blood pressure. Am J Hypertens. 1994;7:249-54.

27. Stranges S, Wu T, Dorn JM, Freudenheim JL, Muti P, Farinaro E, et al. Relationship of alcohol drinking pattern to risk of hypertension: a population-based study. Hypertension. 2004;44:813-9.

28. Renaud S, De Lorgeril M. Wine, alcohol, platelets, and the French paradox for coronary heart disease. Lancet 1992;339:1523-6.

29. Criqui MH, Ringel BL. Does diet or alcohol explain the French paradox? Lancet. 1994;344:1719-23

30. Zilkens RR, Burke V, Hodgson JM, Barden A, Beilin LJ, Puddey IB. Red wine and beer elevate blood pressure in normotensive men. Hypertension. 2005;45:874-9.

31. Opie LH, Lecour S. The red wine hypothesis: from concepts to protective signalling molecules. Eur Heart J. 2007;28:1683-93.
32. Barefoot JC, Gronbaek M, Feaganes JR, McPherson RS, Williams RB, Siegler IC. Alcoholic beverage preference, diet, and health habits in the UNC Alumni Heart Study. Am J Clin Nutr. 2002;76:466-72.

33. Alcacera MA, Marques-Lopes I, Fajo-Pascual M, Foncillas JP, Carmona-Torre F, Martinez-Gonzalez MA. Alcoholic beverage preference and dietary pattern in Spanish university graduates: the SUN cohort study. Eur J Clin Nutr. 2008;62:1178-86.

34. Gronbaek M, Becker U, Johansen D, Gottschau A, Schnohr $\mathrm{P}$, Hein HO, et al. Type of alcohol consumed and mortality from all causes, coronary heart disease, and cancer. Ann Intern Med. 2000;133:411-9.

35. Kristal AR, Peters U, Potter JD. Is it time to abandon the food frequency questionnaire? Cancer Epidemiol Biomarkers Prev. 2005;14:2826-8. 